\title{
Scintillator-based diagnostic for fast ion loss measurements on DIII-D
}

Cite as: Rev. Sci. Instrum. 81, 10D307 (2010); https://doi.org/10.1063/1.3490020

Submitted: 13 May 2010 . Accepted: 30 July 2010 . Published Online: 12 October 2010

R. K. Fisher, D. C. Pace, M. García-Muñoz, W. W. Heidbrink, C. M. Muscatello, M. A. Van Zeeland, and Y. B. Zhu

\section{ARTICLES YOU MAY BE INTERESTED IN}

Scintillator based detector for fast-ion losses induced by magnetohydrodynamic instabilities in the ASDEX upgrade tokamak

Review of Scientific Instruments 80, 053503 (2009); https://doi.org/10.1063/1.3121543

Conceptual design of the ITER fast-ion loss detector

Review of Scientific Instruments 87, $11 D 829$ (2016); https://doi.org/10.1063/1.4961295

Basic physics of Alfvén instabilities driven by energetic particles in toroidally confined plasmas Physics of Plasmas 15, 055501 (2008); https://doi.org/10.1063/1.2838239
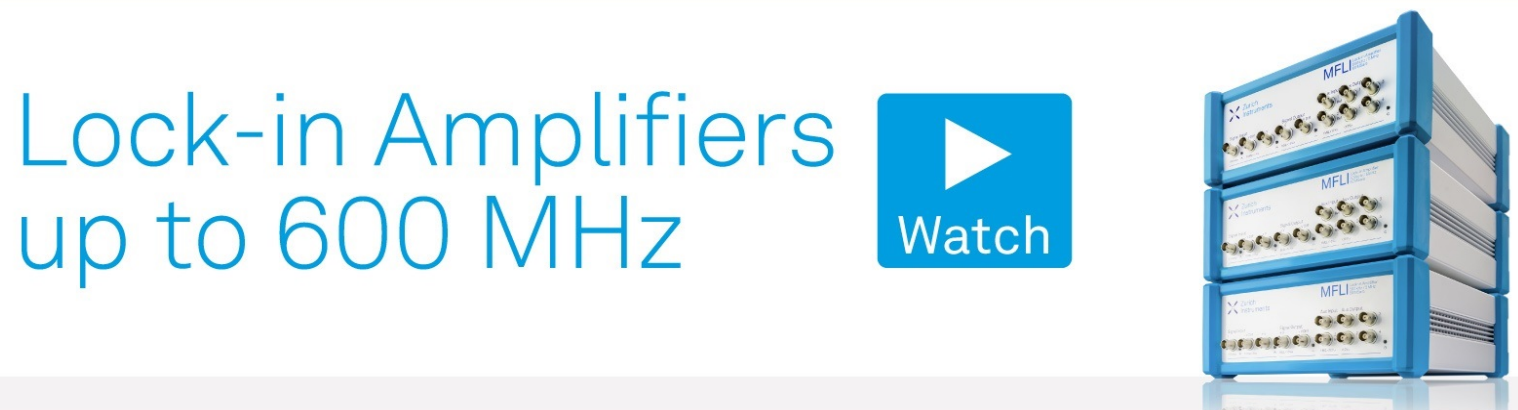

Rev. Sci. Instrum. 81, 10D307 (2010); https://doi.org/10.1063/1.3490020

81, $10 D 307$

(c) 2010 American Institute of Physics. 


\title{
Scintillator-based diagnostic for fast ion loss measurements on DIII-D ${ }^{a)}$
}

\author{
R. K. Fisher, ${ }^{1, b)}$ D. C. Pace, ${ }^{2}$ M. García-Muñoz, ${ }^{3}$ W. W. Heidbrink, ${ }^{2}$ C. M. Muscatello, ${ }^{2}$ \\ M. A. Van Zeeland, ${ }^{1}$ and Y. B. Zhu ${ }^{2}$ \\ ${ }^{1}$ General Atomics, P.O. Box 85608, San Diego, California 92186-5608, USA \\ ${ }^{2}$ University of California-Irvine, Irvine, California 92167, USA \\ ${ }^{3}$ Max Planck-Institut für Plasmaphysik, Garching D-85748, Germany
}

(Presented 17 May 2010; received 13 May 2010; accepted 30 July 2010; published online 11 October 2010)

\begin{abstract}
A new scintillator-based fast ion loss detector has been installed on DIII-D with the time response $(>100 \mathrm{kHz})$ needed to study energetic ion losses induced by Alfvén eigenmodes and other MHD instabilities. Based on the design used on ASDEX Upgrade, the diagnostic measures the pitch angle and gyroradius of ion losses based on the position of the ions striking the two-dimensional scintillator. For fast time response measurements, a beam splitter and fiberoptics couple a portion of the scintillator light to a photomultiplier. Reverse orbit following techniques trace the lost ions to their possible origin within the plasma. Initial DIII-D results showing prompt losses and energetic ion loss due to MHD instabilities are discussed. () 2010 American Institute of Physics.

[doi:10.1063/1.3490020]
\end{abstract}

\section{SUMMARY AND INTRODUCTION}

Fast ions from neutral beam injection, ion cyclotron heating, and fusion reactions play a fundamental role in the heating and stability of tokamak plasmas. Sawteeth and tearing modes affect fast ion confinement, while large fast ion densities can drive collective instabilities, including Alfvén eigenmodes (AEs). The effects of MHD activity on fast ions can significantly impact the plasma performance. Losses of energetic alpha particles from deuterium-tritium fusion in ITER will reduce the alpha heating available to reach ignition and have the potential to cause major damage to the first wall. Given their importance for burning plasma experiments in ITER, fast ion studies have become a high priority area for experimental research on DIII-D.

The internal mode structures of the fast ion induced instabilities inside the DIII-D plasma have been measured using electron cyclotron emission, beam emission spectroscopy, far infrared scattering, reflectometry, and $\mathrm{CO}_{2}$ interferometry measurements. ${ }^{1}$ Measured profiles of the confined beam ions in DIII-D using the recently developed fast ion $D_{\alpha}$ (FIDA) spectroscopy ${ }^{2}$ show that instabilities redistribute fast ions radially outward. Faraday collector-based beam ion loss detectors (BILD) have been used to obtain information on fast ion losses near the outer midplane of DIII-D. ${ }^{3}$

We recently installed a new fast ion loss detector (FILD) on DIII-D. By correlating the beam ion loss results from the new FILD with the other fast ion diagnostics and with observations of the internal mode structures in DIII-D, we gain important information on the fast ion loss orbits and loss mechanisms involved in the instabilities. Using the measured

\footnotetext{
a) Contributed paper, published as part of the Proceedings of the 18th Topical Conference on High-Temperature Plasma Diagnostics, Wildwood, New Jersey, May 2010.

b)Electronic mail: fisherr@fusion.gat.com.
}

pitch angle and energy of the fast ion losses, reverse orbit following techniques can be used to trace the lost ions to their possible origin within the plasma. ${ }^{4}$ The initial results of the FILD diagnostic showing prompt losses and beam ion losses due to MHD instabilities will be discussed.

\section{NEW FAST ION LOSS DETECTOR}

The FILD detector is installed through a radial port located below the outer midplane of DIII-D. As shown in Fig. 1, this detector can be inserted past the first wall tiles and into the region outside the last closed plasma flux surface, which allows the detection of escaping fast ions over a larger portion of phase space than the BILD detectors which are recessed behind the plasma facing the tiles at the outer midplane. The FILD detector measures fast ions that pass through a small entrance aperture in its graphite heat shield and strike a scintillator-coated plate. Measuring the twodimensional light pattern from the scintillator yields information on both the perpendicular gyroradius and the pitch angle of the escaping fast ions, an approach first used to detect escaping fusion products ${ }^{5}$ and more recently used to study losses of energetic ions from rf heating and neutral beam heating. ${ }^{6}$

Figure 2 shows a schematic drawing of the FILD diagnostic. The detector head has a graphite heat shield (diameter $\sim 8.9 \mathrm{~cm}$ ) to protect the detector from the plasma and beam ion heat loads. Thermocouples located just inside the graphite heat shield are used to monitor the temperature of the detector head. The scintillator plate is mounted on the backside of the graphite end cap so that it can be viewed through a vacuum window at the opposite end of the FILD. The ion entrance aperture is $\sim 15 \mathrm{~mm}$ from the plane of the scintillator. The aperture is oriented to accept ions traveling in the same direction as the plasma current. The line from the center of the aperture to the center of the scintillator lies $\sim 45^{\circ}$ 


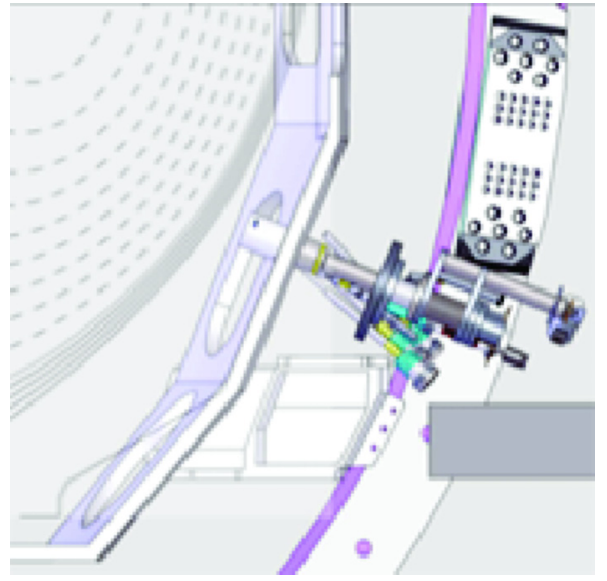

FIG. 1. (Color online) FILD detector is inserted into the region below the midplane of DIII-D and outside the last closed plasma flux surface.

above the direction of the total magnetic field. Based on the ASDEX-Upgrade (AUG) design and orbit modeling calculations, the entrance aperture is $1.1 \mathrm{~mm} \times 3 \mathrm{~mm}$ to provide adequate gyroradius and pitch angle resolution, respectively. The calculated gyroradius resolution of the FILD is $\sim 1.4 \mathrm{~cm}$ for the full energy component, while the pitch angle resolution is $\sim 6^{\circ}$ at a gyroradius of $2.5 \mathrm{~cm}$. Choosing a smaller aperture would have improved the resolution, but would have reduced the FILD signal level and made it more difficult to study high frequency instabilities. Light from the scintillator is collected by a $5 \mathrm{~cm}$ diameter objective lens with a focal length of $60 \mathrm{~cm}$ that is located $60 \mathrm{~cm}$ from the scintillator. The spatial distribution of the scintillation light is imaged using a PCO Pixelfly VGA CCD camera with a Navitar f/N1.8 zoom lens, which has a focal length range of $10-110 \mathrm{~mm}$. The camera and zoom lenses are mounted to the back of the FILD diagnostic just behind a vacuum window and move radially with the insertion of the detector head, so that the optical views remain constant. The camera frame rate is adjustable and provides a maximum sampling of

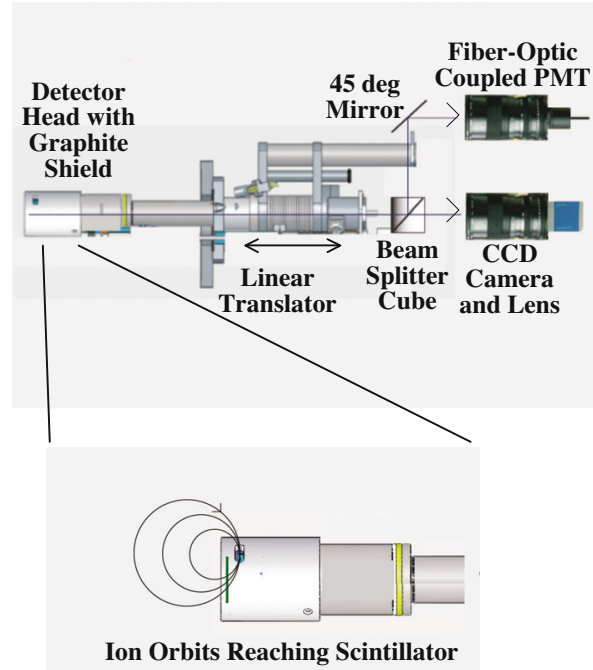

FIG. 2. (Color online) Light from the FILD scintillator is imaged onto camera and fiber-coupled PMT using beam splitter, mirror, and two zoom lenses mounted just behind the vacuum window (Ref. 7).

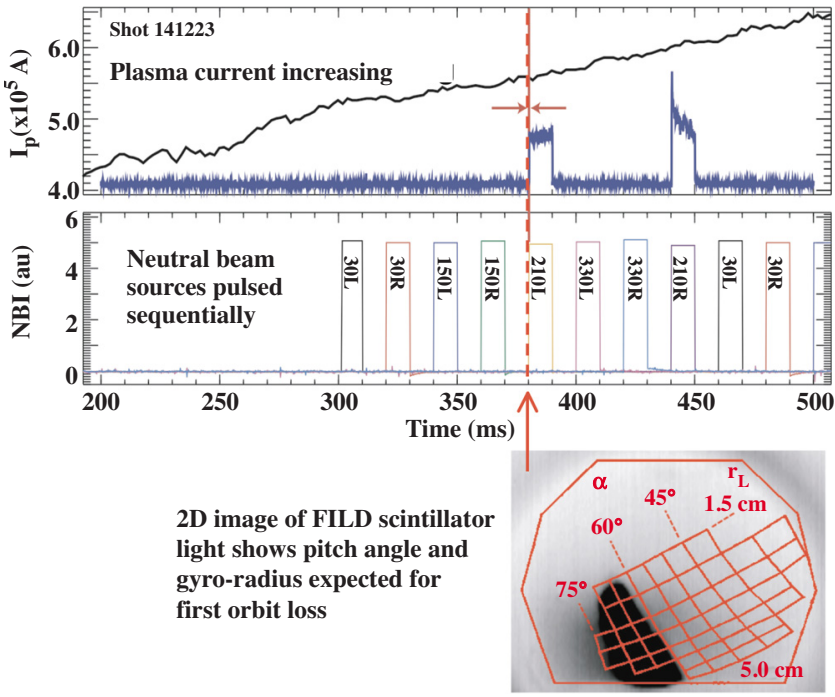

FIG. 3. (Color online) First orbit losses were observed during pulsed operation of neutral beam sources while slowly increasing plasma current (Ref. 7).

$\sim 160 \mathrm{~Hz}$. The Pixelfly camera is located very close to DIII-D but has not required shielding against magnetic fields or against neutron and gamma-ray radiation. We have not observed any signs of radiation damage after several months of operating the Pixelfly camera in the neutron and gammaray environment on DIII-D. We did observe the typical neutron or gamma-ray induced noise in the form of individual saturated pixels during the plasma discharge.

The TG-Green scintillator, first used on the AUG fast ion loss detector, ${ }^{6}$ has a 490 ns decay time, high light generation (ionoluminescense) efficiency, and high saturation levels. For fast time response measurements, a beam splitter cube couples $\sim 50 \%$ of the scintillation light to a second $10-110 \mathrm{~mm}$ zoom lens designed to image the entire scintillator onto the input of a single $1500 \mu \mathrm{m}$ diameter fiber. This single fused silica fiber couples the scintillation light to a Hamamatsu H5783 photomultiplier tube. The PMT output current is amplified by an Advance Research Instruments PMT-4V3 fast amplifier, operating at a gain of $0.1 \mathrm{~V} / \mu \mathrm{A}$. The resulting signal is then digitized at $1 \mathrm{MHz}$, allowing measurements of the beam ion losses with the time response $(>100 \mathrm{kHz})$ and detection sensitivity needed to study Alfvén eigenmodes and other high frequency MHD instabilities.

\section{EXPERIMENTAL RESULTS}

The FILD diagnostic has been installed and operating for approximately 5 months of DIII-D operation. First orbit losses have been used to test the FILD capabilities and performance. Pulsing the neutral beam sources while slowly ramping up the plasma current allows observation of the first orbit losses from each of the beam lines. Figure 3 shows that large first orbit losses are observed from the two ion sources (210L and $210 \mathrm{R})$ that inject through a port near to the $225^{\circ}$ toroidal location of the FILD. A camera image of the twodimensional scintillation light pattern on the scintillator is shown in the lower portion of Fig. 3. The red grid lines show 

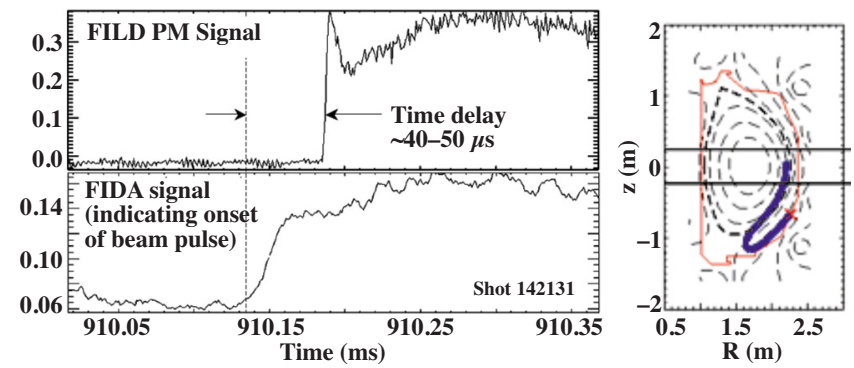

FIG. 4. (Color online) The measured loss signal is delayed by $\sim 40-50 \mu$ s, as expected based on the transit time for first orbit losses (Ref. 7).

the calculated pitch angle and gyroradius based on the detector geometry, aperture location, and magnetic equilibrium. First orbit losses from 210L neutral beam appear at a pitch angles $\sim 70^{\circ}$, in agreement with the reverse orbit calculations for this detector location. ${ }^{4}$ For the $210 \mathrm{R}$ injection at $440 \mathrm{~ms}$, we observe losses at a pitch angle of $\sim 55^{\circ}$, again in agreement with our reverse orbit calculations. The total magnetic field at the detector is $\sim 1.5 \mathrm{~T}$, so that the corresponding gyroradii for the full, one-half, and one-third energy components of the $75 \mathrm{keV}$ deuterium beams are $\sim 3.8,2.7$, and $2.2 \mathrm{~cm}$, respectively. The gyroradius resolution of the FILD is on the order of $1.4 \mathrm{~cm}$ for the full energy component. The light pattern on the scintillator shown in Fig. 3 appears consistent with the energies and pitch angles expected for first orbit losses from the 210L beam.

The FILD PMT signal measures the time dependence of the fast ion loss signal on a microsecond time scale. Figure 4 shows the PMT signal and one of the signals from the FIDA diagnostic as a function of time near the start of one of the beam pulses from the 210R beamline on DIII-D. The FIDA signal is the $D_{\alpha}$ light resulting from confined beam ions interacting with the $210 \mathrm{R}$ beam injected neutrals. Hence, the onset of the FIDA signal indicates the start of the 210R beam pulse. The onset of the FILD PMT signal is delayed by 40-50 $\mu$ s, which is consistent with the transit time for the beam ions over the prompt loss orbit, shown on the right side of Fig. 4, calculated by reverse orbit following techniques. ${ }^{4}$ The red X marking the end of the orbit indicates the FILD location.

Beam ion losses have also been observed as a result of a number of MHD instabilities, including neoclassical tearing modes, $q=2$ fishbones, and AE activity. Figure 5 shows the measured beam ion loss signals during AE activity increased significantly once the FILD entrance aperture was inserted past the front face of the plasma tiles, but then remained nearly constant as the detector head was inserted further into the region outside the last closed plasma flux surface. As shown in Fig. 1, the detector head always remains at least $10 \mathrm{~cm}$ outside of the last closed flux surface at this port location below the plasma midplane.

Because the detector head is located near one of the

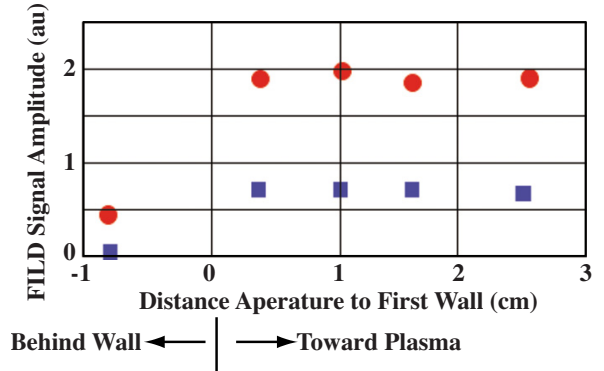

FIG. 5. (Color online) Measured loss signals from the 210R (red circles) and $210 \mathrm{~L}$ (blue squares) during $\mathrm{AE}$ activity were nearly constant once the ion entrance aperture was inserted past the front face of the plasma wall tiles (Ref. 7).

counterinjected neutral heating beams on DIII-D, we observed a large prompt loss signal due to this beam. The large flux of energetic beam ions resulting from the first orbit losses caused local scintillator damage, as evidenced by a drop in the scintillator emission in subsequent shots. The scintillator region near $70^{\circ}$ pitch angle and $3.5-4 \mathrm{~cm}$ gyroradius (lower portion of bright region on the left in Fig. 3) showed the largest damage.

Despite this limitation, the fast response and high scintillation efficiency of the TG-Green scintillator allows measurements at the high frequencies of interest for many MHD instabilities, including Alfvén eigenmodes. The observed losses resulting from neoclassical tearing modes, $q=2$ fishbones, and AE activity are still being analyzed and will be discussed in subsequent papers in our efforts to better understand how the instabilities interact with the fast ions.

\section{ACKNOWLEDGMENTS}

This work was supported in part by the U.S. Department of Energy under Grant Nos. DE-FC02-04ER54698, SCG903402, and DE-FG03-94ER54271.

${ }^{1}$ M. A. Van Zeeland, G. J. Kramer, M. E. Austin, R. L. Boivin, W. W. Heidbrink, M. A. Makowski, G. R. McKee, R. Nazikian, W. M. Solomon, and G. Wang, Phys. Rev. Lett. 97, 135001 (2006).

${ }^{2}$ W. W. Heidbrink, N. N. Gorelenkov, Y. Luo, M. A. Van Zeeland R. B. White, M. E. Austin, K. H. Burrell, G. J. Kramer, M. A. Makowski, G. R. McKee, and R. Nazikian, Phys. Rev. Lett. 99, 245002 (2007).

${ }^{3}$ Y. B. Zhu, W. W. Heidbrink, and L. D. Pickering, Nucl. Fusion 50, 084024 (2010).

${ }^{4}$ D. C. Pace, R. K. Fisher, M. Garcia-Munoz, D. S. Darrow, W. W. Heidbrink, C. M. Muscatello, R. Nazikian, M. A. Van Zeeland, and Y. B. Zhu, Rev. Sci. Instrum. 81, 10D305 (2010).

${ }^{5}$ S. J. Zweben et al., Nucl. Fusion 30, 1551 (1990); D. Darrow, S. Baeumel, E. Cecil et al., Rev. Sci. Instrum. 77, 10E701 (2006).

${ }^{6}$ M. García-Muñoz, H.-U. Fahrbach, and H. Zohm, Rev. Sci. Instrum. 80, 053503 (2009); A. Werner, A. Weller, and D. S. Darrow, ibid. 72, 780 (2001); K. Shinohara, M. Isobe, and D. S. Darrow, ibid. 77, 10E521 (2006); D. Darrow, ibid. 79, 023502 (2008).

${ }^{7}$ R. K. Fisher, D. C. Pace, M. García-Muñoz, R. L. Boivin, E. D. Frederickson, W. W. Heidbrink, C. M. Muscatello, R. Nazikian, C. C. Petty, M. A. Van Zeeland, and Y. B. Zhu, "Measurements of beam ion losses on DIII-D due to MHD instabilities," Proceedings of the 37th EPS Conference on Plasma Physics, Dublin, Ireland, 2010. 